\title{
QUADRATIC FORMS OVER COMPLETE LOCAL RINGS
}

\author{
Manuel Ojanguren and Raman Parimala
}

\section{INTRODUCTION}

Let $A$ be a complete excellent local domain of Krull dimension 2 and $K$ its field of fractions. We further assume that 2 is invertible in $A$ and that the residue field of $A$ is algebraically closed. We first show that the unramified Brauer group of $K$ (with respect to all discrete valuations of $K$ ) vanishes. Using this result we prove that every rank 4 quadratic form which is isotropic in all completions of $K$ with respect to discrete valuations, is isotropic. For $K=\mathbb{C}((x, y))$ this was announced by P. Jaworski in 1999, at a conference on quadratic forms in Oberwolfach. The results presented here, obtained immediately after the conference, are a consequence of our efforts to give a short proof of Jaworski's announcement. They subsequently led to various generalizations, notably in the case when the residue field of $A$ is real closed (see [1] and, for further developments, [2] and [3]). Jaworski's proof has now appeared as well [6].

\section{The UNRAMIFIED BRAUER GROUP}

Let $A$ be as above and $m$ its maximal ideal. Let $\pi: X \rightarrow \operatorname{Spec}(A)$ be a desingularization [8] of $\operatorname{Spec}(A)$. Since $X$ is obtained from $\operatorname{Spec}(A)$ by a sequence of blowing ups and normalizations, the map $\pi$ is proper. We denote by $X_{n}$ the fibre of $\operatorname{Spec}\left(A / m^{n+1}\right)$.

We prove a result (Lemma 2.2) in the spirit of Lemma 3.3 of [5].

Lemma 2.1. The natural maps

$$
\operatorname{Pic}\left(X_{n}\right) \rightarrow \operatorname{Pic}\left(X_{n+1}\right)
$$

are surjective.

Proof. This follows from the exact sequence of sheaves

$$
0 \longrightarrow \frac{m^{n} \mathcal{O}_{X}}{m^{n+1} \mathcal{O}_{X}} \longrightarrow\left(\frac{\mathcal{O}_{X}}{m^{n+1} \mathcal{O}_{X}}\right)^{*} \longrightarrow\left(\frac{\mathcal{O}_{X}}{m^{n} \mathcal{O}_{X}}\right)^{*} \longrightarrow 1,
$$

noting that

$$
H^{2}\left(X, \frac{m^{n} \mathcal{O}_{X}}{m^{n+1} \mathcal{O}_{X}}\right)=H^{2}\left(X_{0}, \frac{m^{n} \mathcal{O}_{X}}{m^{n+1} \mathcal{O}_{X}}\right)=0
$$

because $X_{0}$ is of dimension 1 .

Lemma 2.2. The canonical homomorphism

$$
\operatorname{Br}(X) \rightarrow \lim _{\leftarrow} \operatorname{Br}\left(X_{n}\right)
$$

is injective.

Proof. Let $\mathcal{A}$ be an Azuamya algebra over $X$. Denote by $\mathcal{A}_{n}$ the algebra obtained from $\mathcal{A}$ under base change from $X$ to $X_{n}$ and suppose that it is trivial for each $n$. Let

$$
u_{n}: \mathcal{A}_{n} \stackrel{\sim}{\rightarrow} \mathcal{E} n d\left(V_{n}\right)
$$

be an isomorphism, where $V_{n}$ is a locally free sheaf on $X_{n}$. The sheaf $V_{n}$ is determined by $\mathcal{A}_{n}$ up to a line bundle. By Lemma 2.1 we can successively modify each $V_{n}$ in such a way that $V_{n}$ is isomorphic to $V_{n+1} \otimes_{\mathcal{O}_{X_{n+1}}} \mathcal{O}_{X_{n}}$. In this case the isomorphisms $u_{n}$ also form a projective system. By [5], 5.1.4, the projective system $\left(V_{n}, n \in \mathbb{N}\right)$ gives a locally free $\mathcal{O}_{X}$-module $V$ and an isomorphism

$$
u: \mathcal{A} \stackrel{\sim}{\rightarrow} \mathcal{E} n d(V) .
$$


Corollary 2.3. The Brauer group of $X$ is trivial. In particular, the unramified Brauer group of $K$ is trivial.

Proof. In fact, since $X_{n}$ is a curve over $\operatorname{Spec}\left(A / m^{n+1}\right)$ and $A / m$ is algebraically closed, $B r\left(X_{n}\right)=0$ (See [5], page 101). By a well-known purity theorem ([4], Proposition 2.3) an unramified element of $B r(K)$ is in the image of $\operatorname{Br}(X) \rightarrow \operatorname{Br}(K)$ and hence is zero.

\section{QUADRATIC FORMS}

Theorem. Let $A$ be a complete excellent local domain of Krull dimension 2 and $K$ its field of fractions. Assume that 2 is invertible in $A$ and that the residue field of $A$ is algebraically closed. Every rank 4 quadratic form $q$ over $K$ which is isotropic over every completion of $K$ at a discrete valuation, is isotropic.

Proof. After scaling we may assume that $q=<1, a, b, a b d>$ with $a, b, d \in K^{*}$. If $d$ is a square, then $q$ is the norm form of the quaternion algebra $\mathcal{A}=\left(\frac{a, b}{K}\right)$. The condition that $q$ is isotropic at all completions implies that $\mathcal{A}$ is split at all completions of $K$. In particular $\mathcal{A}$ is unramified in $\operatorname{Br}(K)$ and hence, by 2.3 , is zero. In particular, $q$ is hyperbolic.

Suppose now that $d$ is not a square. Let $L=K(\sqrt{d})$. The field $L$ satisfies the same assumptions as $K$. The form $q_{L}$ over $L$ has trivial discriminant and is isotropic at all completions of $L$ at discrete valuations. By the previous case, $q_{L}$ is hyperbolic. The form $q$ therefore contains a multiple of $<1,-d>([7]$, Ch. 7, Lemma 3.1) and, being of discriminant $d$, also contains a subform of discriminant 1 . Hence $q$ is isotropic.

\section{REFERENCES}

1. J.-L. Colliot-Thélène, M. Ojanguren and R. Parimala, Quadratic forms over fraction fields of two-dimensional henselian rings and Brauer groups of related schemes, Proceedings of the International Colloquium on 'Algebra, Arithmetic \& Geometry' held at T.I.F.R., Mumbai, 2000.

2. J.-L. Colliot-Thélène, Ph. Gille et R. Parimala, Arithmétique des groupes algébriques linéaires sur certains corps géométriques de dimension deux, C. R. Acad. Sci. Paris 333, Série I (2001), 827-832.

3. J.-L. Colliot-Thélène, Ph. Gille and R. Parimala, Arithmetic of linear algebraic groups over two-dimensional geometric fields, preprint (2002).

4. A. Grothendieck, Le groupe de Brauer II, Dix exposés sur la cohomologie des schémas, North Holland, 1968.

5. A. Grothendieck, Le groupe de Brauer III, Dix exposés sur la cohomologie des schémas, North Holland, 1968.

6. P. Jaworski, On the strong Hasse principle for fields of quotients of power series rings in two variables., Math. Z. 236 (2001), 531-566.

7. T.Y. Lam, Algebraic theory of quadratic forms, Benjamin, 1973.

8. J. Lipman, Rational singularities with appications to algebraic surfaces and unique factorization, Publ. Math. IHES 36 (1969), 195-280.

Manuel O janguren, Institut de mathématiques, University of Lausanne, Ch-1015 Lausanne, Shitzerland

Raman Parimala, School of Mathematics, Tata Institute of Fundamental Research, Homi Bhabha Road, M UMBAI, INDIA 